\title{
MEGARA spectrograph for the GTC: mechanical and opto- mechanical design
}

\author{
Maldonado-Medina, Manuel *a , García-Vargas, María Luisa ${ }^{\mathrm{a}}$, Gil de Paz, Armando , Sánchez- \\ Blanco, Ernesto ${ }^{a}$, Pérez-Calpena, Ana ${ }^{\mathrm{a}}$, Gallego Maestro, Jesús ${ }^{\mathrm{b}}$, Carrasco Licea, Esperanza ${ }^{\mathrm{c}}$, \\ Sánchez Moreno, Francisco ${ }^{\text {d }}$, Vílchez Medina, José ${ }^{\mathrm{e}}$ \& MEGARA Team \\ ${ }^{\mathrm{a}}$ FRACTAL SLNE (Madrid, Spain), ${ }^{\mathrm{b}}$ Universidad Complutense de Madrid (Madrid, Spain), \\ ${ }^{\mathrm{c}}$ INAOE (Puebla, México), ${ }^{\mathrm{d}}$ Universidad Politécnica de Madrid (Madrid, Spain), ${ }^{\mathrm{e}}$ Instituto de \\ Astrofísica de Andalucía IAA-CSIC (Granada, Spain)
}

\begin{abstract}
MEGARA (Multi-Espectrógrafo en GTC de Alta Resolución para Astronomía) is the future optical Integral-Field Unit (IFU) and Multi-Object Spectrograph (MOS) for the GTC $10.4 \mathrm{~m}$ telescope. This contribution summarizes the current mechanical design of the spectrograph and the adopted solutions for the mechanisms and the opto-mechanical components.
\end{abstract}

Keywords: MEGARA, GTC, mechanical design, mechanism, spectrograph, opto-mechanics, mechanism

\section{INTRODUCTION}

MEGARA (Multi-Espectrógrafo en GTC de Alta Resolución para Astronomía) is a fiber-fed spectrograph with two optical Integral-Field Units (IFU) and a set of robotic positioners for Multi-Object Spectroscopy (MOS) that will be installed at Folded-Cassegrain focus of the GTC (Gran Telescopio CANARIAS) 10.4-m telescope. The fibers will feed one (MEGARA-Basic) or two (MEGARA-Advanced) spectrographs to be placed on a Nasmyth-type platform.

The MEGARA IFU mode will offer two different bundles, The MEGARA IFU mode will offer two different bundles, one covering 12.5" x 11.3" with a spaxel size of 0.62" (Large Compact Bundle; LCB) and another one covering 8.5" x 6.7" with a spaxel size of 0.42 " (Small Compact Bundle;SCB). The MEGARA MOS will allow observing up to 100 objects thanks to the MEGARA MOS positioners that can place a mini-bundle of 7 fibers ( 0.62 "/fiber) covering an area of 1.6" on the sky in a region of 3.5' x 3.5' around the two IFU bundles. Eight of these bundles will be devoted to the determination of the sky during the observation with the LCB IFU.

The IFUs and the 100 positioners that constitute the Fiber MOS shall be installed at a GTC Folded Cassegrain focal station. Then, IFU and MOS fibers bundles shall be routed through the telescope structure and rotators to the Spectrograph entrance.

This paper describes the current mechanical design of the spectrograph and the adopted solutions for the mechanisms and the opto-mechanical components. MEGARA Preliminary Design Review took place on 21-22 March 2012.

*manuel.maldonado@fractal-es.com

Ground-based and Airborne Instrumentation for Astronomy IV, edited by lan S. McLean, Suzanne K. Ramsay, Hideki Takami, Proc. of SPIE Vol. 8446, 844655 - ( ) 2012 SPIE · CCC code: 0277-786X/12/\$18 - doi: 10.1117/12.925754 


\section{OVERALL DESCRIPTION OF THE SPECTROGRAPH}

In MEGARA Spectrograph the optical elements are placed on an optical table. Apart from the "baseline" optical elements, such as the collimator and the camera, there is a mechanism that automatically interchanges 11 volume phase holograms (VPHs), a mechanism that provides focus adjustment, another mechanism that allows the interchange of fiber bundles, and a rotating shutter.

The baseline optical train is shown on Fig.1 and the overall view of the spectrograph is shown in Fig.2.

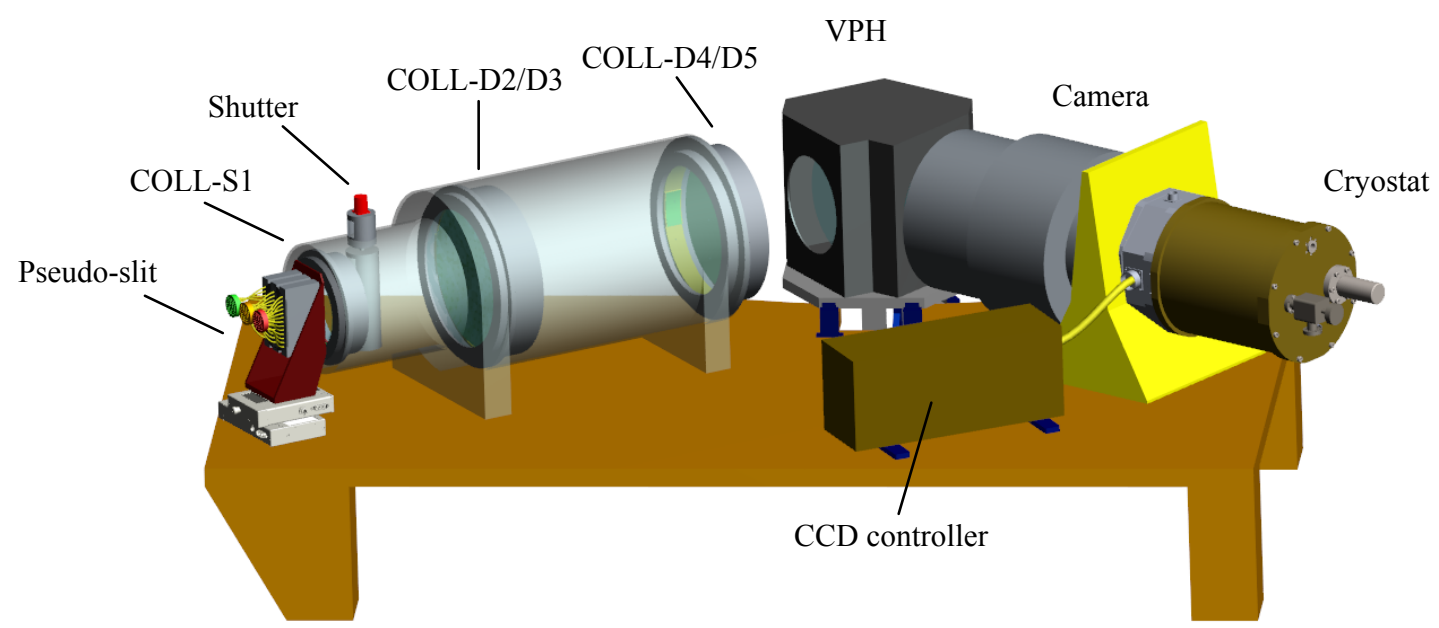

Fig. 1. CAD model of the baseline optical train of MEGARA spectrograph

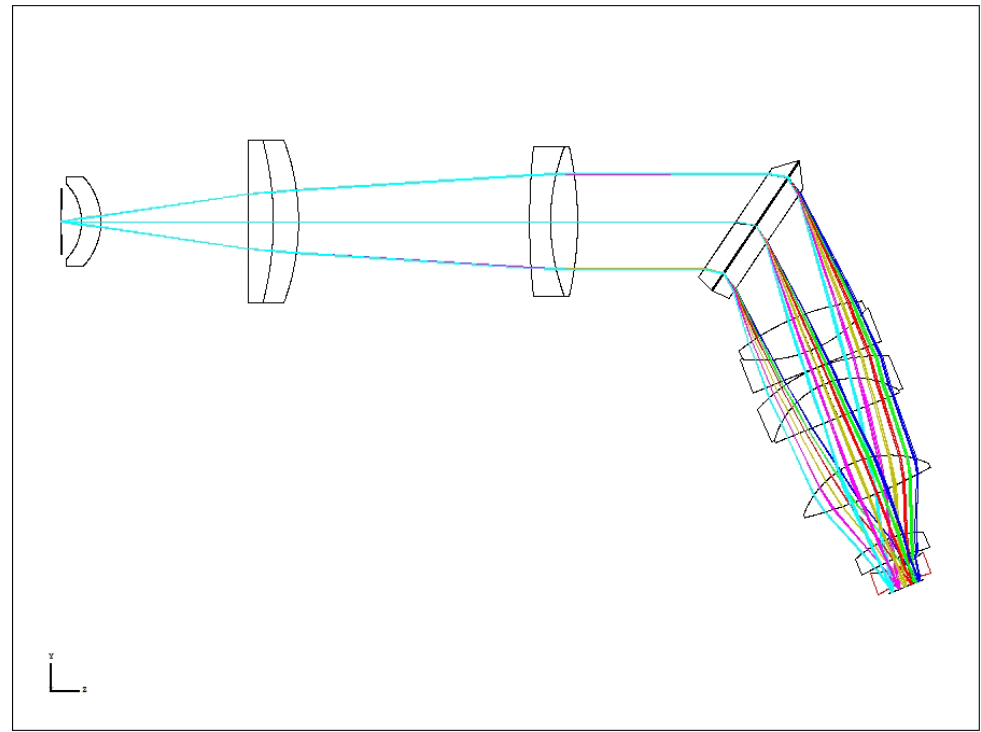

Fig. 2. MEGARA spectrograph raytracing of one of the VPH gratings 


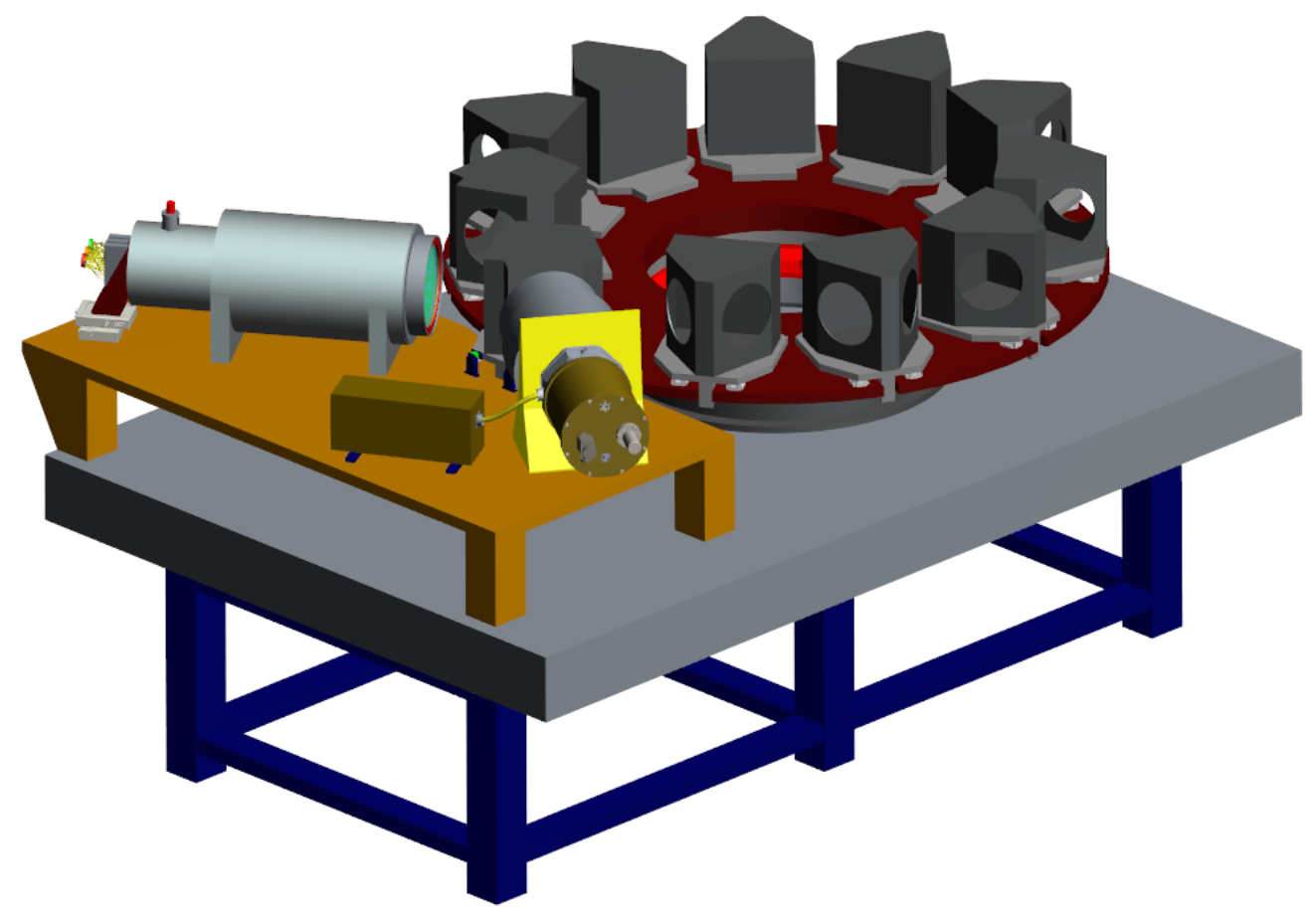

Fig. 3. MEGARA spectrograph complete view

The fiber bundles reach the spectrograph on the pseudo-slit, where the fibers are aligned. The mount for the first collimator lens is placed just behind the pseudo slit. Follows the shutter, which is a rotating custom-made shutter.

As established by on the optical design, it is desirable to allow a little focus adjustment. In this design that is done by moving the pseudo-slits, mounting its support structure on a linear commercial translation stage.

The camera is attached to the same plate that the CCD head in order to minimize differential rotations between them. Then, this plate is joined with a specific support to the optical table. The CCD controller is placed close to the CCD head to minimize cable length.

\section{SPECTOGRAPH MECHANICS SUBSYSTEMS}

This section is devoted to provide a description of the main MEGARA spectrograph subsystems, which includes (a) the optical bench and auxiliary table, (b) the pseudo slit arrangement and mechanisms, (c) Collimator barrel and shutter (d) the VPH gratings wheel, (e) the VPH insertion mechanism elements, (f) the wheel drive

\subsection{Optical bench}

The whole spectrograph is located on a $2 \mathrm{~m} \times 3 \mathrm{~m} \times 0,2 \mathrm{~m}$ optical table. As a standard size, this table could be ordered from one of the main manufacturers of optical tables (Newport, Melles Griot, Thorlabs, Kinetic, etc.)

All optical elements but the gratings wheel are located in another table that is $290 \mathrm{~mm}$ above the main table. This avoids having large optical mounts for the lenses. The size of this table is $900 \times 1750 \mathrm{~mm}$. There is also a small platform $(200 \mathrm{x}$ $280 \mathrm{~mm}$ ) that is used to support the gratings insertion mechanism. On the right side it's a little bit larger to accommodate 
the pseudo-slit translation stage. The table is supported by a rigid carbon steel truss structure, designed to interface with the telescope instruments platform.

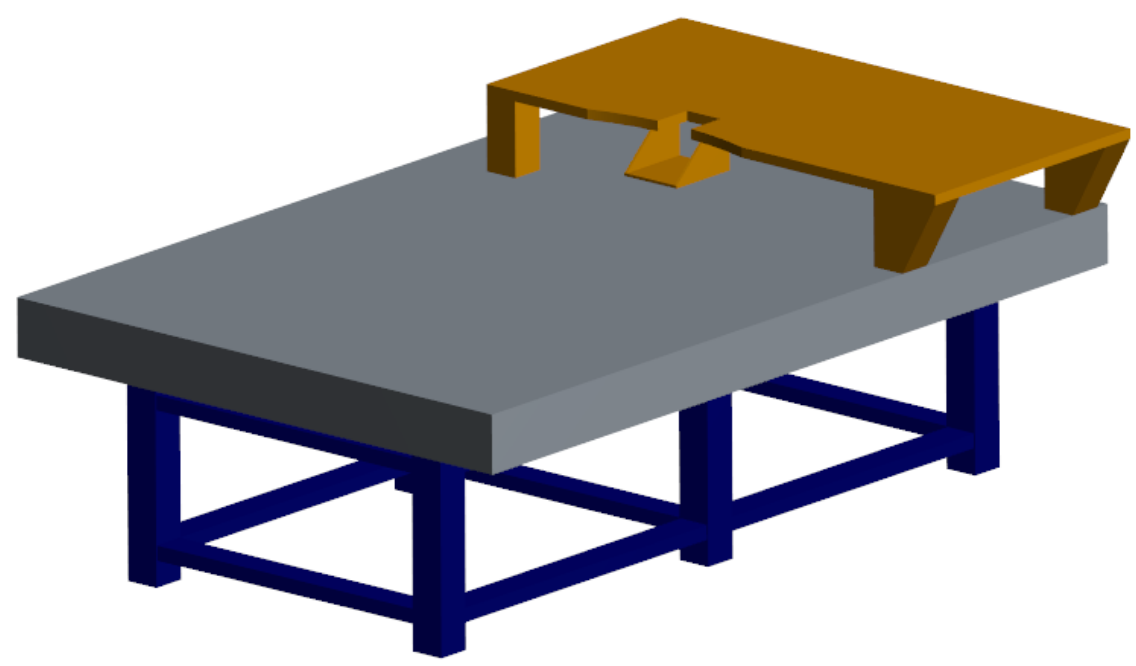

Fig. 4. Main optical table with auxiliary platform

\subsection{Pseudo slit arrangement}

The fibers are aligned in a pseudo-slit at the spectrograph entrance. In the scenario of "MEGARA Basic" there is only one spectrograph for 3 fiber bundles. Two bundles are coming from the central IFUs (LCB and SCB) and the other comes from the robotic positioners. There must be is a "switching" system to select one another. Figure 6 shows the system, where three different fiber bundles are simulated with tubes of different colors.

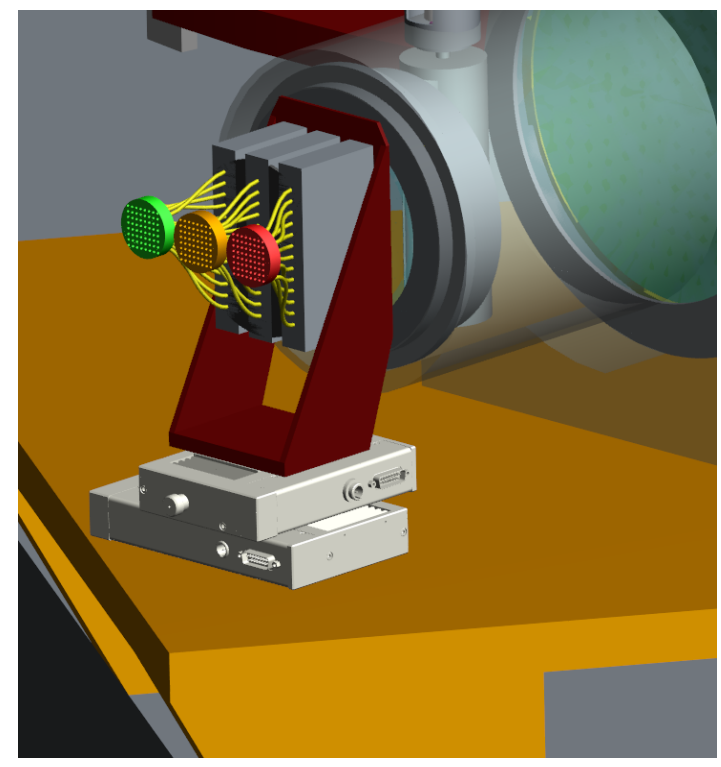

Fig. 5. Close-up view of the pseudo-slit support structure on a pair of orthogonal translation stages. 
The selection of the light source in the pseudo-slit is done placing the pseudo-slits on a structure that is on a translation stage that locates in the center of the optical system the fibers in use. The light coming for the other fibers is blocked with a baffle that is at the front of the first lens of the collimator, which is placed right after the pseudo-slit.

The translation stage proposed is a commercial component, which features all in one base, table, motor, precision recirculating ballscrew, preloaded nuts, reference and limit switches.

The chosen linear positioning stage comes from Physik Instrumente model M-605 (see Figure 8). This stage has a travel range of $50 \mathrm{~mm}$, the minimum incremental motion is $0.3 \mu \mathrm{m}$, the repeatability is $0.1 \mu \mathrm{m}$, the load capacity $300 \mathrm{~N}$. Position reference sensors are located in the middle of the operating range and can be used to reference the absolute position of the stage within $0.5 \mu \mathrm{m}$ accuracy.

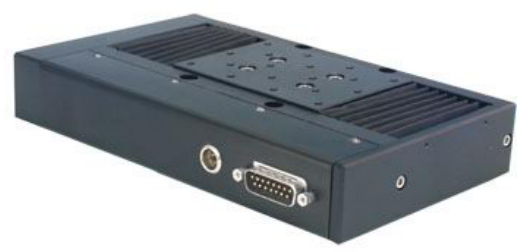

Fig. 6. Physik Instrumente M-605 precision translation stage

We are now evaluating other compact $\mathrm{X}-\mathrm{Y}$ mechanisms. The space savings is clear, but we are also considering reliability parameters.

\section{Focus}

A focusing mechanism has been incorporated in order to allow fine focusing for improving image quality in the different grating configurations. There is another translation stage for the focus adjustment. Its displacement is orthogonal to the previous mechanism, on the direction of the optical axis. Both are stacked, being these stages compatible with this configuration.

The focus depth implies that the minimum step for adjustment shall be $10 \mu \mathrm{m}$ and the total range of movement $\sim 3.5 \mathrm{~mm}$.

\subsection{Collimator barrel and shutter}

The collimator lenses are placed on a common barrel, as seen on figures 1 and 2 . The singlet and doublets are inserted with subcells on this barrel.

The shutter is placed after the first lens of the collimator, inserted in a hole made on the collimator barrel.

It is a rotary shutter with a mini-motor that rotates a cylinder. This cylinder is mechanized to allow the light to be blocked or to pass at $90^{\circ}$ intervals.

By rotating the motor, the cylinder covers completely the slit location. By rotating $90^{\circ}$, the cylinder allows light passing by. The aperture time is defined by adjusting the rotation speed of the motor. Once the shutter is in position the motor must be switched off in order to avoid thermal dissipation

The shutter can be seen on figure 1 inserted on the barrel. Its basic operational principle is sketched on figure 7 . 

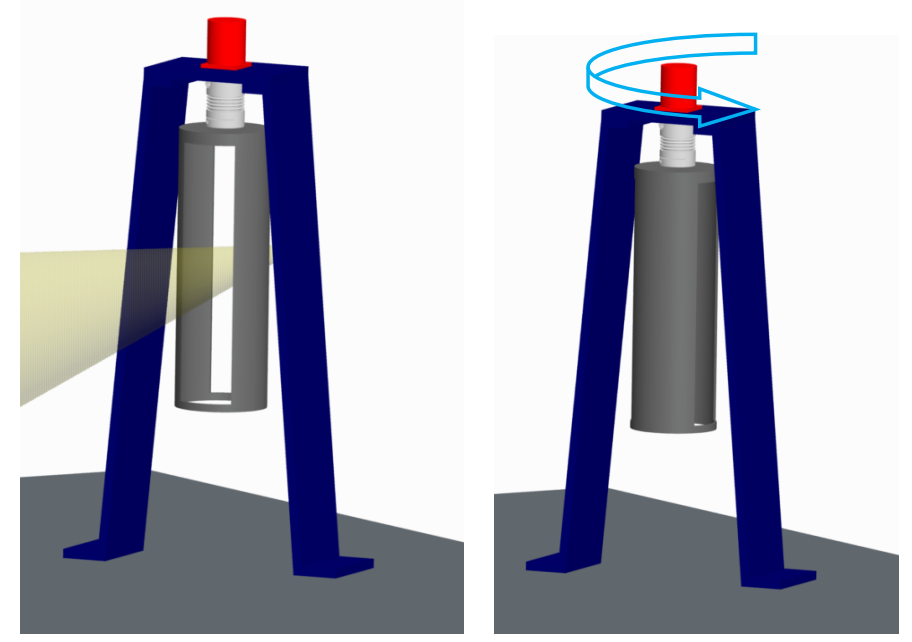

Fig. 7. Rotating shutter. By rotating the motor, the cylinder covers completely the slit location. By rotating $90^{\circ}$, the cylinder allows light passing by.

Next figure shows an example of the second collimator doublet subcell preliminary design. Each mount will be comprised of: A barrel or cell, a flexible aluminum ring, a elastomer O-ring and a screwed blocking ring
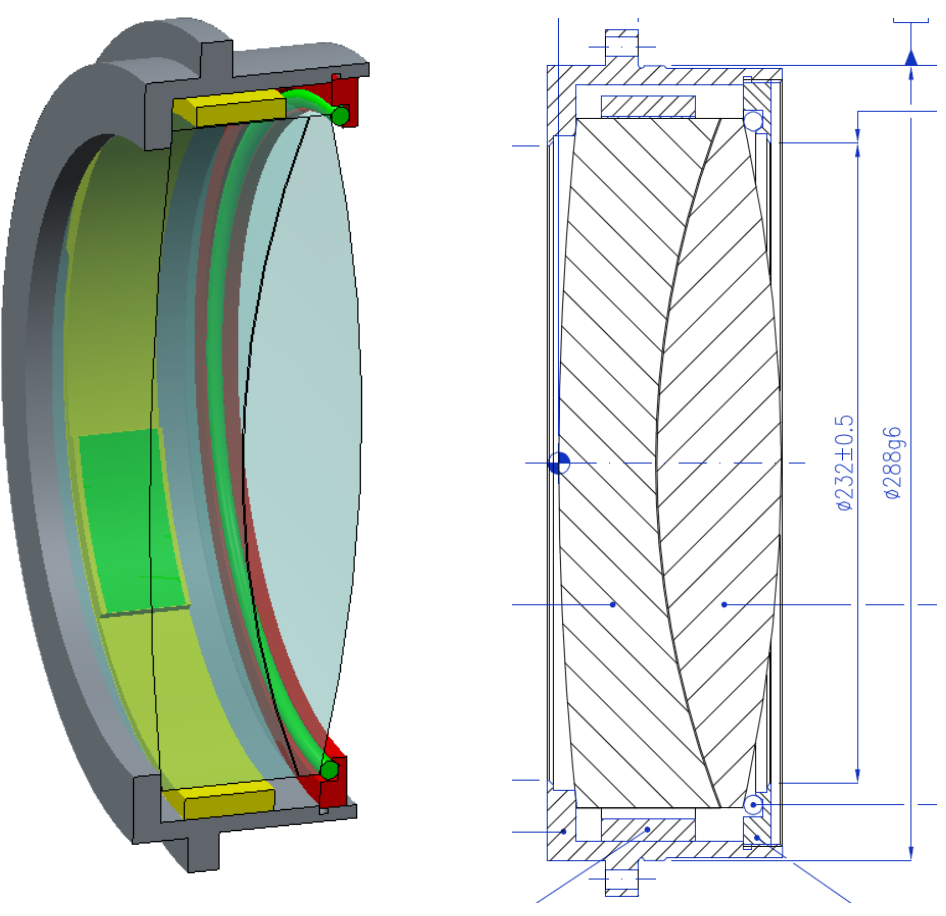

Fig. 8. Opto-mechanical design of a collimator doublet subcell.

\subsection{VPH gratings wheel}

One of the main challenges of this design has been the gratings mechanism. The spectrograph includes the capability of the automatic interchange of $11 \mathrm{VPH}$ gratings that are placed on a large aluminum alloy wheel. Its diameter is Ø1880 
$\mathrm{mm}$. As it shown in the figures, we identify the wheel itself and the VPH mounts located on several platforms. There are 5 low resolution gratings (LR), 4 mid-resolution gratings (MR) and 2 high resolution gratings (HR). It is also possible to locate only MR and HR gratings.

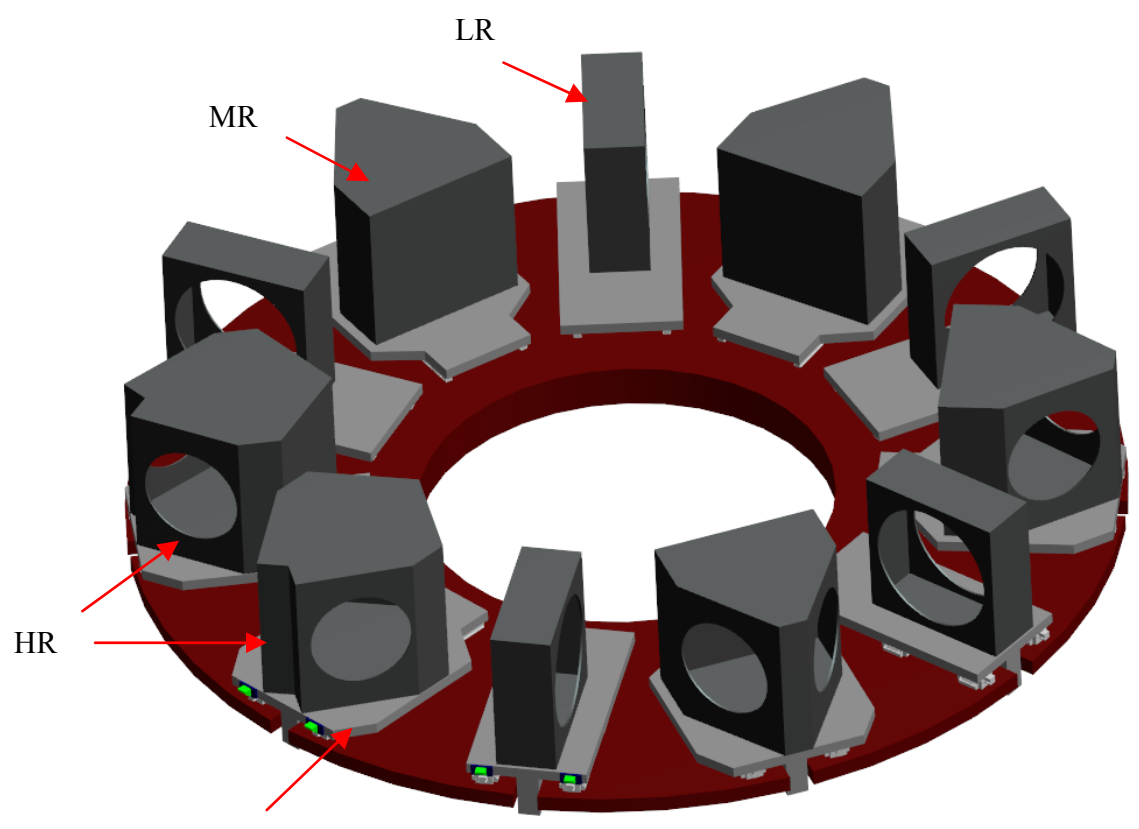

platform

Fig. 9. Detail of VPH gratings wheel with $5 \mathrm{LR}+4 \mathrm{MR}+2 \mathrm{HR}$ mounts

The mechanical mount of each VPH is screwed to the platform. The platform has a pair of guideways screwed on its downside. These guideways are inserted on their corresponding carriages that are screwed to the wheel. It is thus a very rigid assembly.

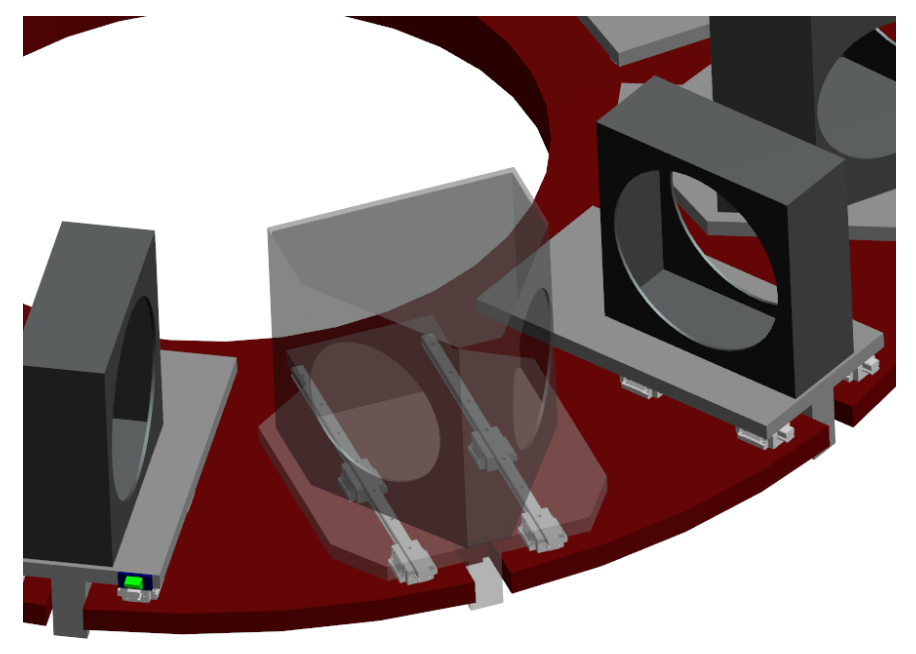

Fig. 10. The VPH platform placed on the railways, and those inserted on their carriages. 
The mount shall be optically aligned with respect to the platform during integration. That position shall be then fixed with reference pins. Should the VPH be removed from the platform, it returns to its exact position when is installed again.

VPH mounts are to be seated in a flat-cone-groove kinematic system with fine alignment capabilities on the platform.

These mounts have not been detailed yet.

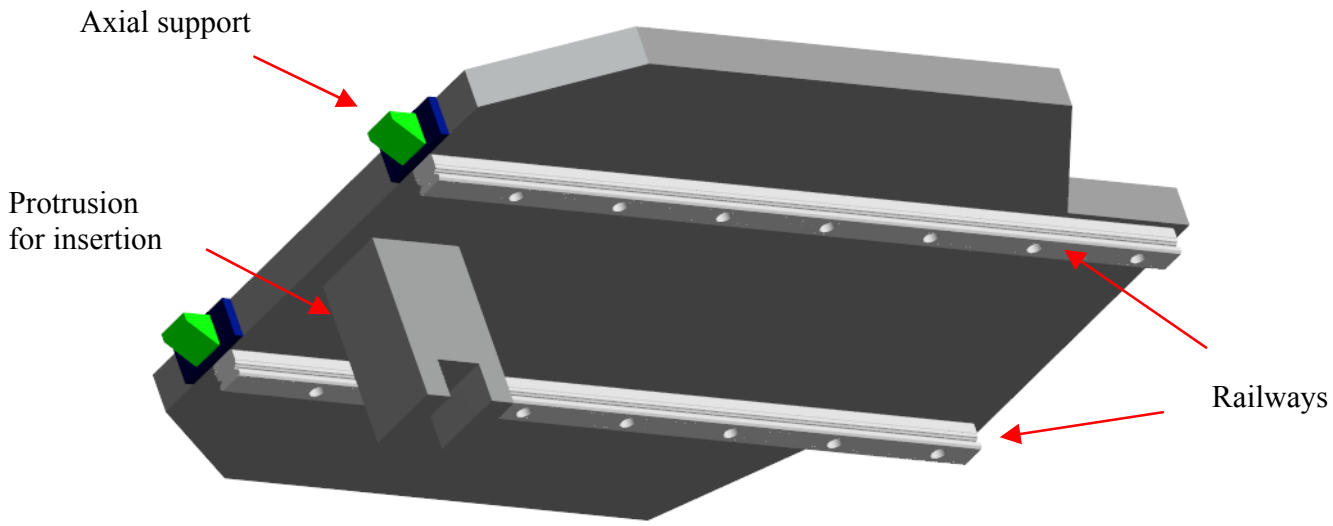

Fig. 11. VPH platform with its characteristic parts.

\subsection{VPH gratings insertion mechanism}

When the wheel has selected the VPH of interest, it is translated to the optical path. The insertion mechanism consists of an electro-mechanical actuator that translates a stud type track roller that moves on a railway. This track roller engages the protrusion of the VPH mount platform and pushes it to the optical path or pulls it out of it, it depends on the situation.

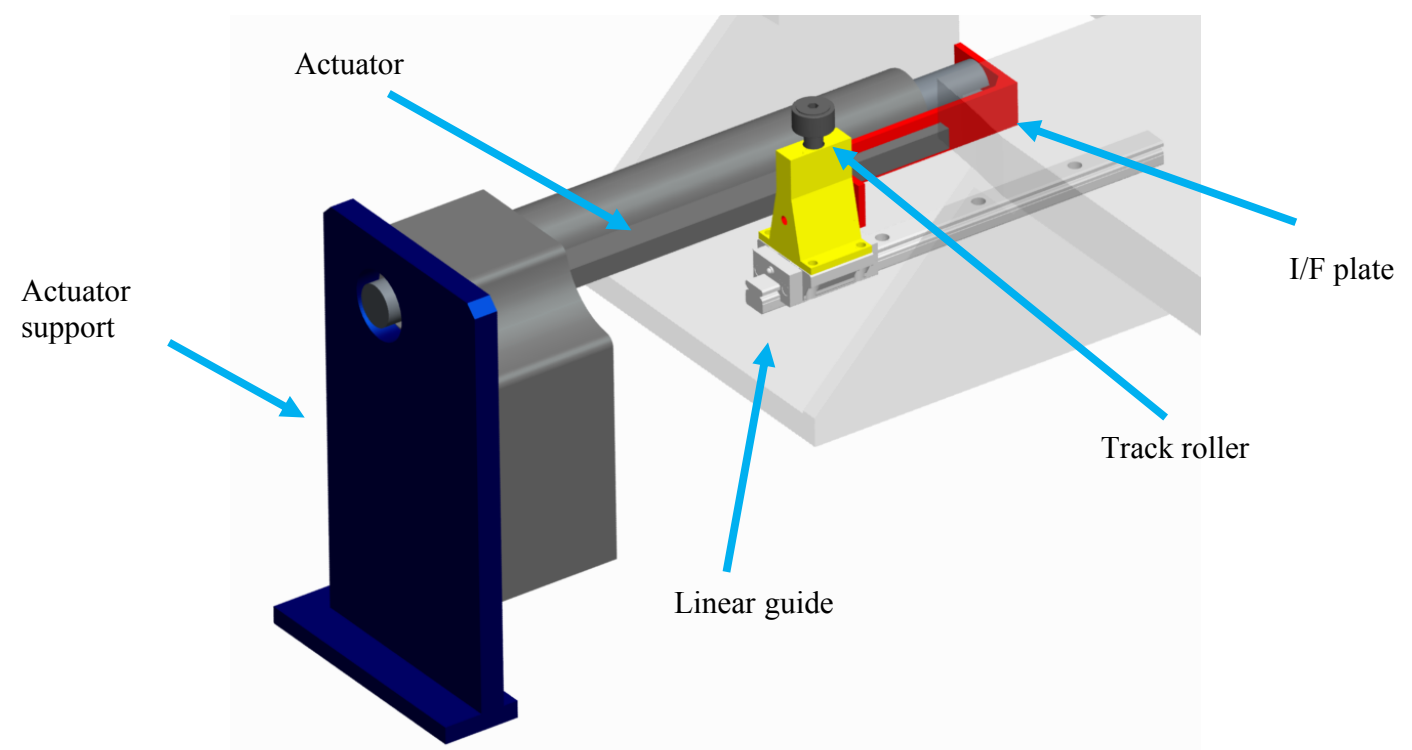

Fig. 12. Functional elements of the insertion mechanism. 

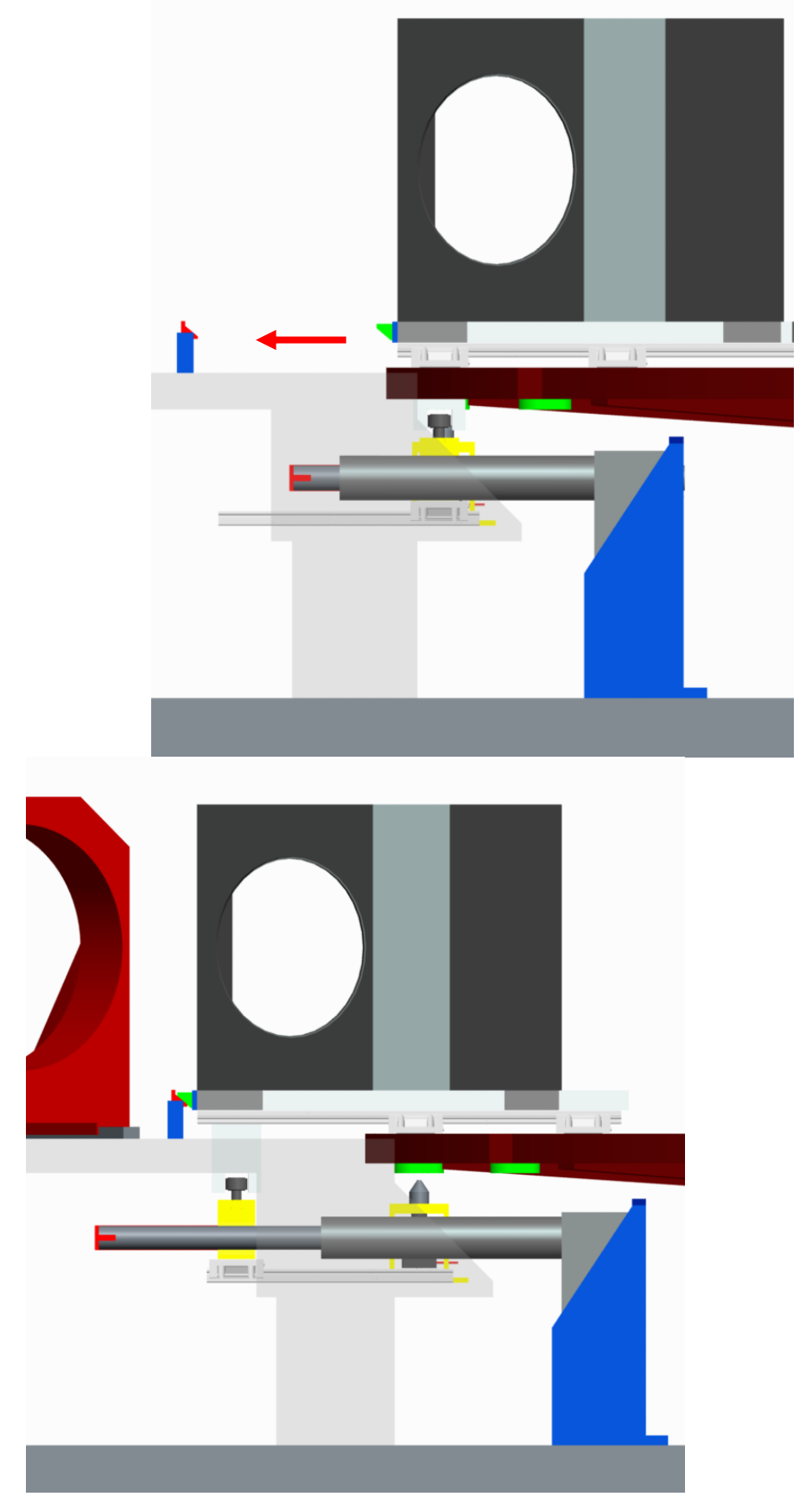

Fig. 13. Sequence of insertion of a HR VPH into the optical path

The elements of the mechanism are identified on next figure. Some elements have been deleted from display to provide a better view of the mechanism.

\author{
Proc. of SPIE Vol. 8446 844655-9
}




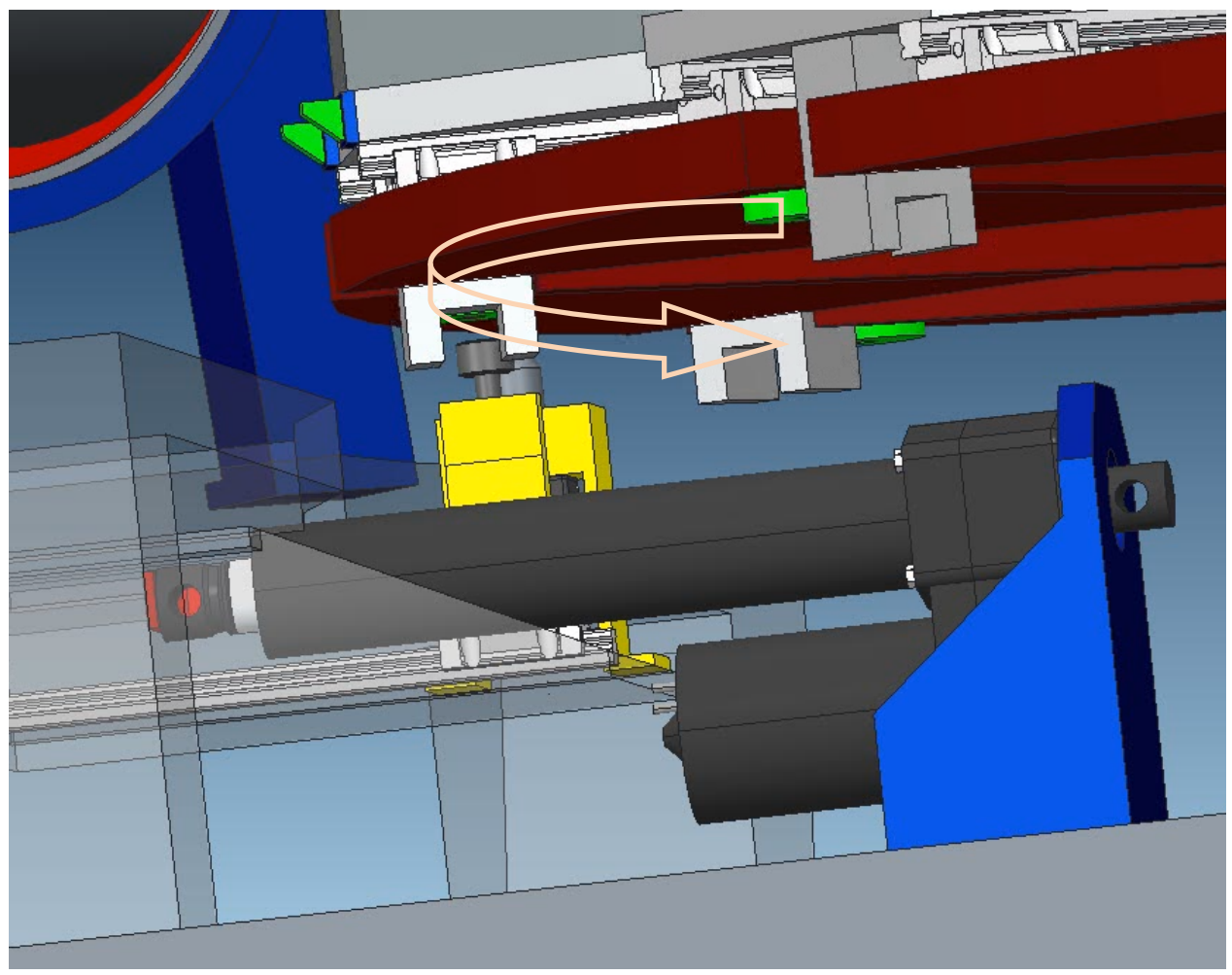

Fig. 14. Platform protrusions are passing by the roller during grating selection

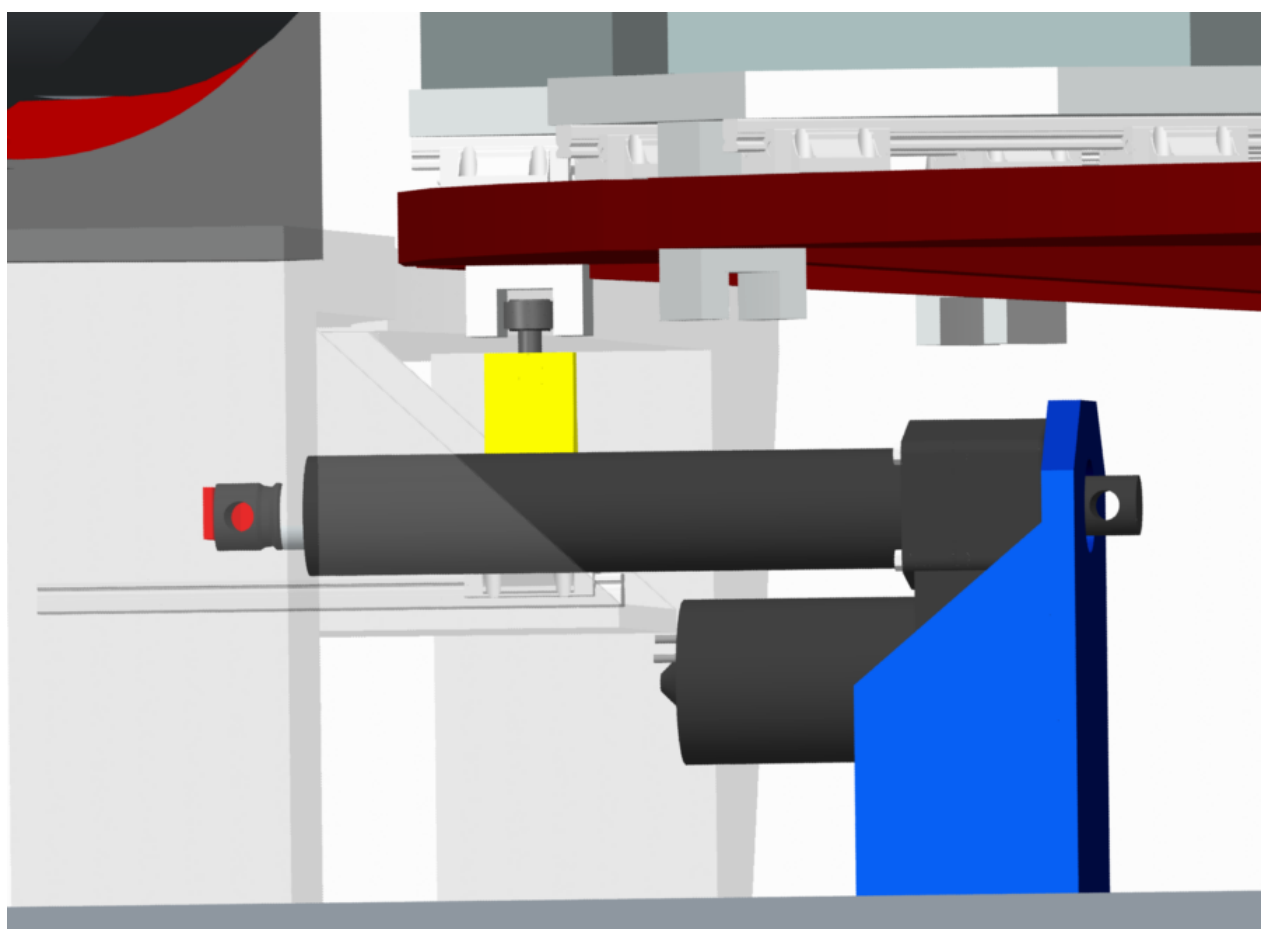

Fig. 15. Another view of the mechanism. Table is transparent. The selected grating is ready to be located on the optical path. 
In order to avoid radial displacements of the VPH gratings during the rotation of the wheel, it is envisaged a delrin circular track, screwed to the table, on which are inserted all the protrusions and with an aperture on the position of the insertion mechanism. At this point it overlaps with the track roller, so the protrusions are secured all the time.

The actuator will be a precision electro-mechanical actuator comprised of a stepper motor, a high precision preloaded ballscrew with an absolute encoder on the motor. This gives a repeatability of $+-0.05 \mathrm{~mm}$. Including the linear guides and the rest of the elements, we are pretty sure that it will achieve a repeatability better than $+-0,1 \mathrm{~mm}$.

The platform will be cantilevered, and the position will be defined by the encoder. There will be a rubber hard stop after the expected end of travel.

The actuators proposed for driving the wheel are pairs of servomotors with planetary reducers preloaded on gears by pinions in order to remove backlash. One motor should have an absolute encoder on axis, as well as the other an incremental encoder. The servo controller monitors the preload. We plan to achieve around 10" of total repeatability for the wheel positioning. These servo motors are on brake when are switched off.

The pre-selected bearing is crossed rolled bearing XSU140844 from INA.

\section{FINITE ELEMENT ANALYSIS}

The scope of the analysis performed is to calculate the displacement of the optical elements of the instrument produced by gravitational strain and thermal strain. The results obtained are then introduced in the optical design in order to analyze their effect on the image motion and image quality of the spectrograph.

A global FE model is used to calculate the gravitational and thermal strain. This model represents the complete spectrograph. It has been simplified to reduce the complexity of the model and the calculations.

Control nodes are placed at the positions of the optical elements in order to obtain their displacements. Point mass are placed at the location of different elements to simulate its weight. These elements are connected to the structure using rigid (not flexible) ideal bars made of the material with the same CTE as the part of the structure where they are attached. It is specified a contact area between the structures and the supporting parts.

The number of elements is 52021, with 134798 nodes.

The loads cases are:

- Uniform thermal variation from $20^{\circ} \mathrm{C}$ to $-2^{\circ} \mathrm{C}$

- Standard earth's gravity 


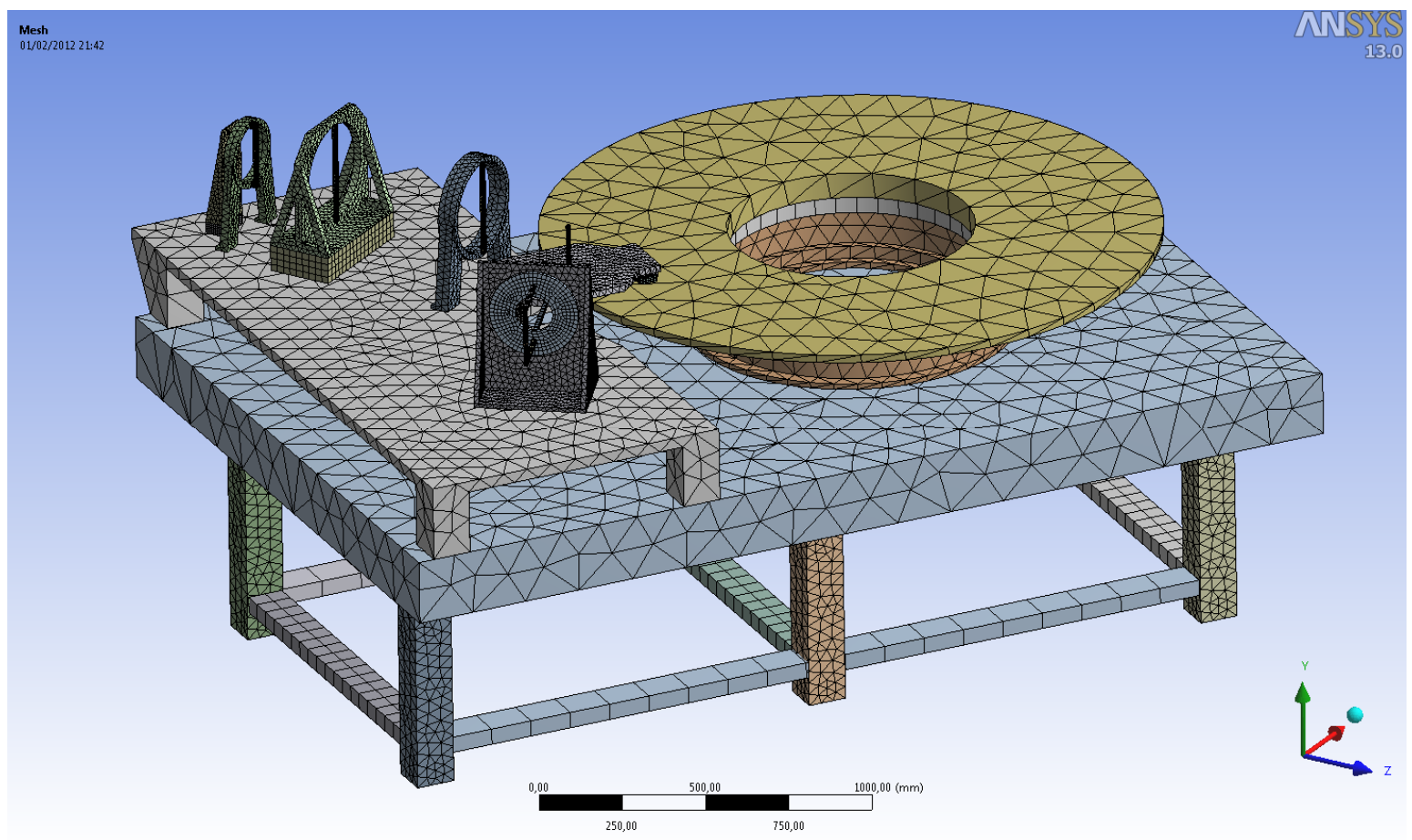

Fig. 16. Finite element model of the spectrograph. In fact, it is a model of a previous version with each collimator lens on an independent support on the table.

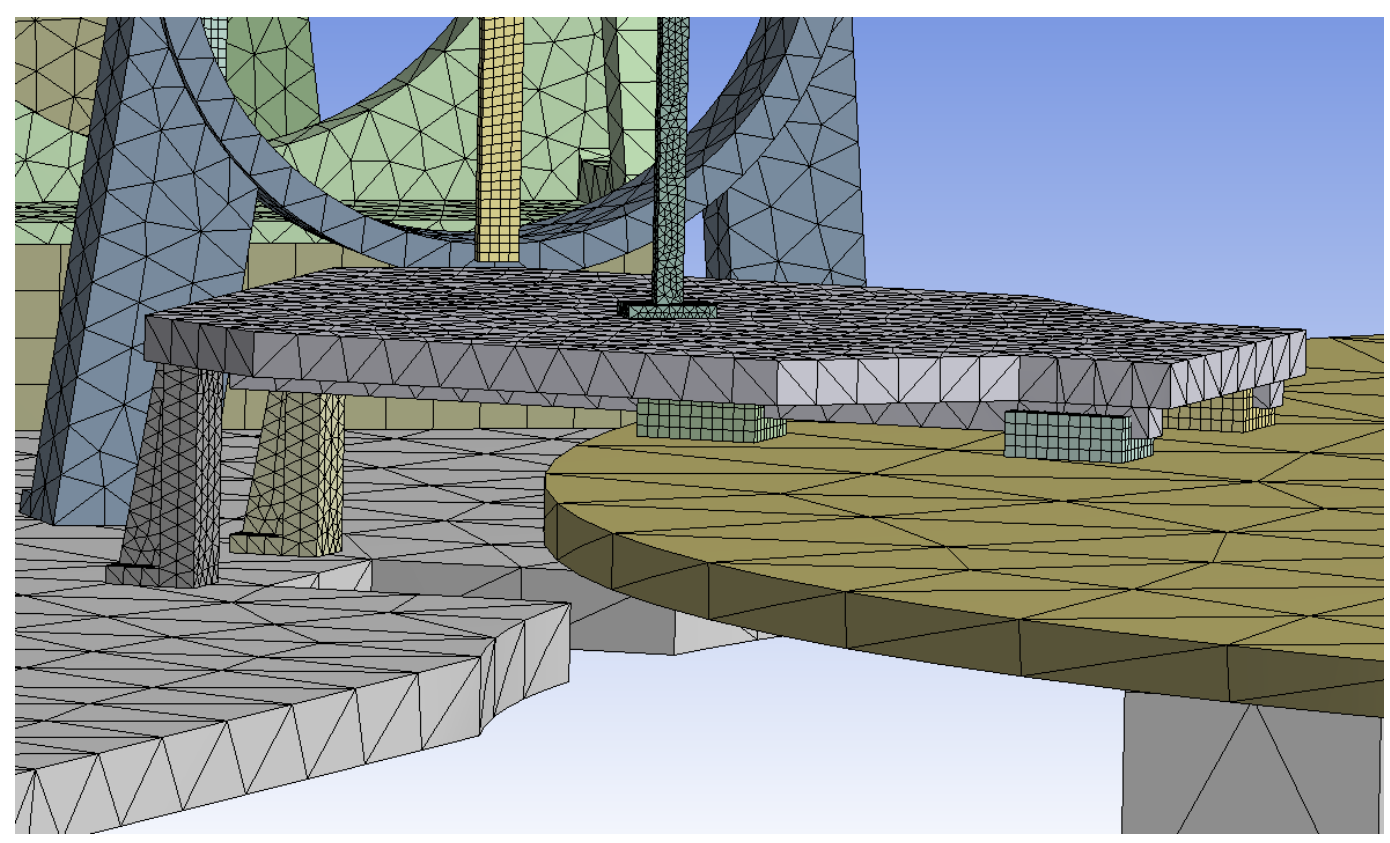

Fig. 17. Detail of the model of the VPH platform 


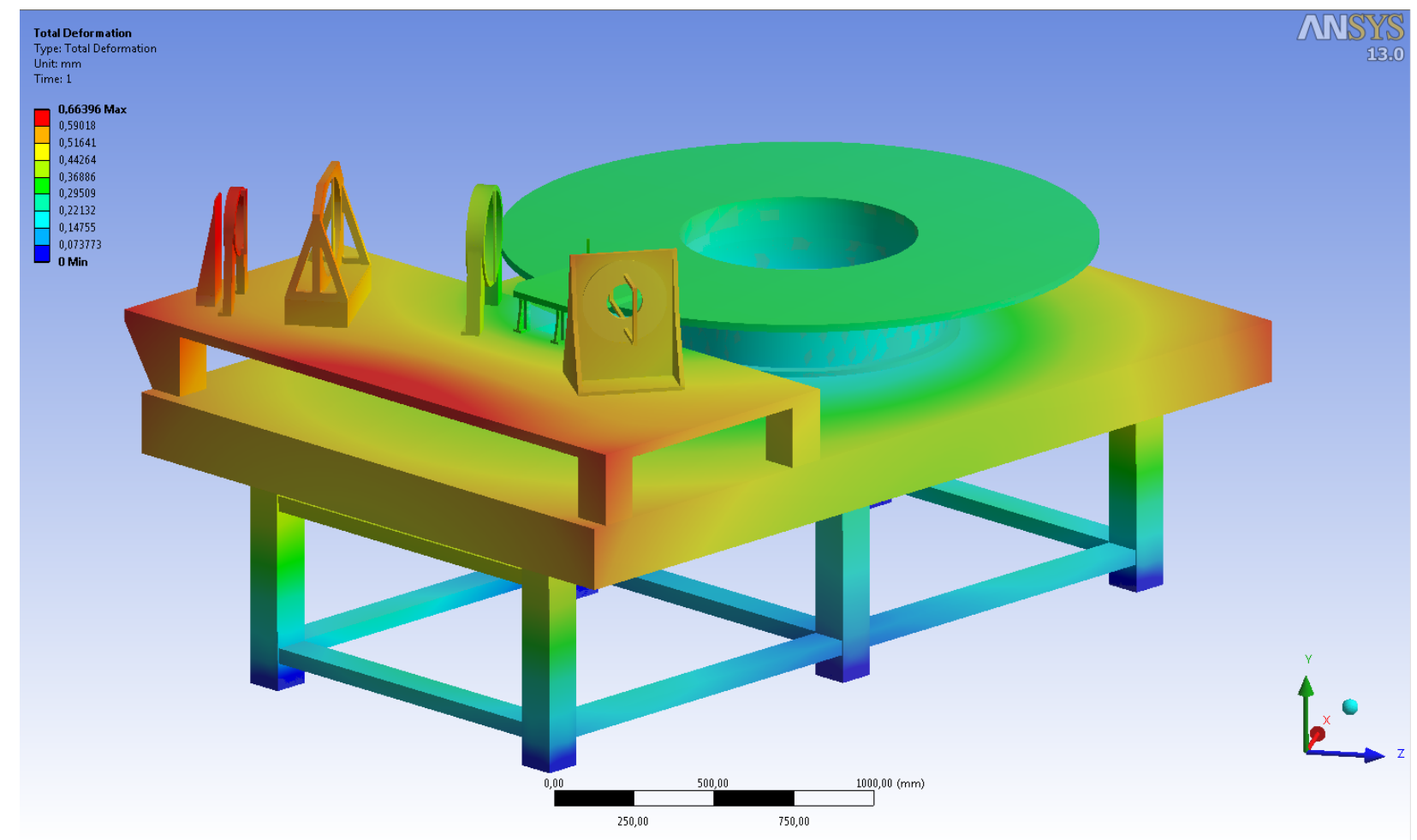

Fig. 18 Total deformation due to gravity and thermal effects

Table 1 summarizes the relative displacements between adjacent control nodes. This gives a first approximation to the structural behavior of the whole spectrograph, in not-yet-optimized design.

\begin{tabular}{|c|c|c|}
\hline Delta X (horizontal decenter) & $(\mathbf{m m})$ & $\operatorname{microns}(\mu \mathrm{m})$ \\
\hline PseudoSlit - COLL-S1 & -0.00288 & -2.88 \\
\hline COLL-S1 - COLL-D2 / COLL-D3 & $-2.11 \mathrm{E}-02$ & -21.065 \\
\hline $\begin{array}{l}\text { COLL-D2 / COLL-D3 - COLL-D4 / } \\
\text { COLL-D5 }\end{array}$ & $-4.95 \mathrm{E}-02$ & -49.493 \\
\hline COLL-D4 / COLL-D5 - Pupil & $+3.77 \mathrm{E}-01$ & +377.332 \\
\hline Pupil - Camera & $+1.79 \mathrm{E}-01$ & +178.553 \\
\hline Camera - Cryostat & $-2.36 \mathrm{E}-02$ & -23.558 \\
\hline Delta Y (vertical decenter) & $(\mathbf{m m})$ & microns $(\mu \mathrm{m})$ \\
\hline PseudoSlit - COLL-S1 & -0.02618 & -26.18 \\
\hline COLL-S1 - COLL-D2 / COLL-D3 & $+6.54 \mathrm{E}-03$ & +6.54 \\
\hline $\begin{array}{l}\text { COLL-D2 / COLL-D3 - COLL-D4 / } \\
\text { COLL-D5 }\end{array}$ & $-8.04 \mathrm{E}-02$ & -80.4 \\
\hline COLL-D4 / COLL-D5 - Pupil & $-3.70 \mathrm{E}-02$ & -37.01 \\
\hline
\end{tabular}




\begin{tabular}{|c|c|c|}
\hline Pupil - Camera & $+1.36 \mathrm{E}-01$ & +136.37 \\
\hline Camera - Cryostat & $-6.94 \mathrm{E}-03$ & -6.94 \\
\hline Delta Z (Focus) & (mm) & microns $(\mu \mathrm{m})$ \\
\hline PseudoSlit - COLL-S1 & +0.04694 & +46.94 \\
\hline COLL-S1 - COLL-D2 / COLL-D3 & $-1.60 \mathrm{E}-01$ & -160.22 \\
\hline $\begin{array}{l}\text { COLL-D2 / COLL-D3 - COLL-D4 / } \\
\text { COLL-D5 }\end{array}$ & $-2.43 \mathrm{E}-01$ & -243.15 \\
\hline COLL-D4 / COLL-D5 - Pupil & $-3.90 \mathrm{E}-02$ & -39.005 \\
\hline Pupil - Camera & $+2.01 \mathrm{E}-01$ & +201.429 \\
\hline Camera - Cryostat & $+1.30 \mathrm{E}-01$ & +130.4 \\
\hline
\end{tabular}

Fig. 19. Relative displacements between control nodes. Give an idea of the global behavior of spectrograph mechanics model.

\section{MEGARA SPECTROGRAPH AT TELESCOPE}

Next figure shows two spectrographs on the Nasmyth platform of the GTC telescope.

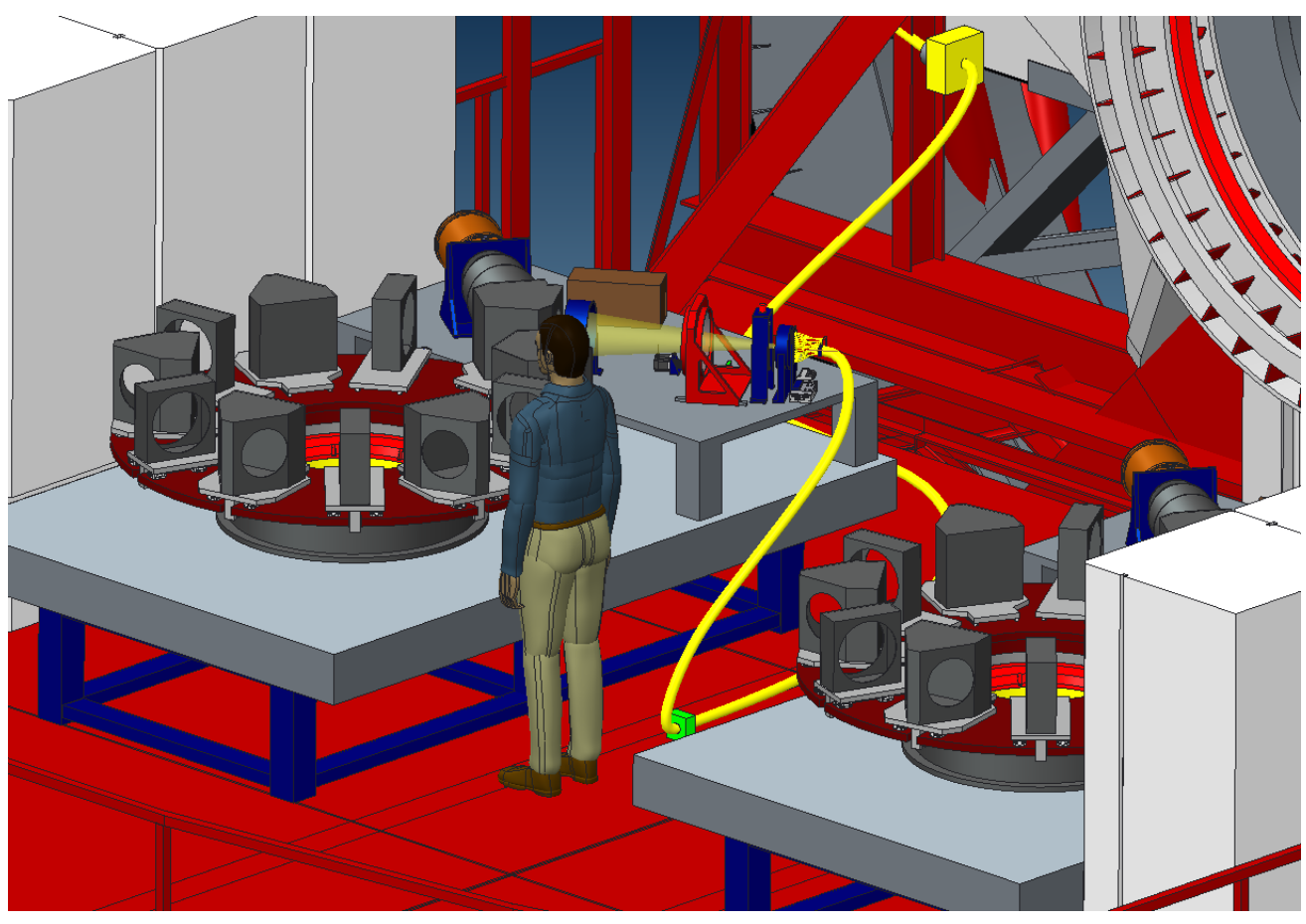

Fig. 20. Partial view of MEGARA at the telescope. An operator is placed to give the sense of scale and an idea of the height of the elements. 


\section{CONCLUSIONS}

The current design of MEGARA spectrograph shows no risks, makes use of proven engineering solutions, provides reliability by using commercial components, allows pseudo-slit exchange, allows VPH exchange and allow focusing to optimize image quality.

The design of the spectrograph mechanical subsystems presented in this paper has already evolved from the design presented at PDR as MEGARA team has included the recommendation agreed with GRANTECAN and the PDR External Reviewers Panel. Now the system is ready to enter into the detailed design.

\section{REFERENCES}

[1] Gil de Paz, Armando et al. "MEGARA: the future optical IFU and multi-object spectrograph for the GTC", These proceedings (8446-182) 